\title{
Ultrasound-Guided Pectoral Nerve Block With Tr Ansverse Thoracic Muscle Plane Block Enhanced World Health Organization on Quality of Life Brief Scale Scores 6 Months After M Odified Radical Mastectomy : A Randomized, Placebo-controlled and Double-Blind Clinical Trial
}

Tianxiao Liu

Guangxi Medical University First Affiliated Hospital

Lina Chen

Guangxi Medical University First Affiliated Hospital

Min Shi

Guangxi Medical University First Affiliated Hospital

Weixin Dai

Guangxi Medical University First Affiliated Hospital

Jing Chen

Guangxi Medical University First Affiliated Hospital

Yubo Xie ( $\square$ xybdoctor@163.com )

Guangxi Medical University First Affiliated Hospital https://orcid.org/0000-0002-7198-7506

Research Article

Keywords: Pectoral nerve block, Transverse thoracic muscle plane block, Quality of Life, Modified radical mastectomy

Posted Date: June 24th, 2021

DOl: https://doi.org/10.21203/rs.3.rs-486686/v1

License: (c) (1) This work is licensed under a Creative Commons Attribution 4.0 International License. Read Full License 


\section{Abstract}

Background Modified radical mastectomy (MRM) has a large incision range and can cause strong intraoperative stress, a high incidence of postoperative acute pain, and chronic pain. There are few studies on the objective evaluation of the perioperative stress response by some stress-related serological indicators and on the long-term follow-up evaluation of postoperative quality of life and the incidence of

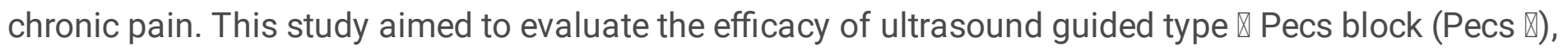

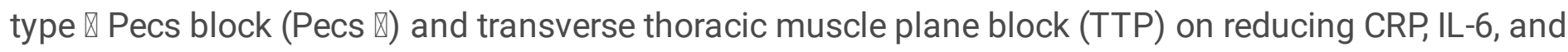
WBC values during surgery and on enhancing World Health Organization on Quality of Life Brief Scale (QOL) scores 6 months after MRM.

Methods The randomized, placebo-controlled and double-blind study was conducted in 76 patients assigned to two groups that received either ultrasound guided Pec $\nabla+P e c \nabla+T T P$ block with $40 \mathrm{ml}$ of $0.25 \%$ ropivacaine (group PT) or saline (group C). The primary outcomes were the changes in CRP, IL- 6 and WBC values on the first day before surgery and the first and third days after surgery, the changes in blood glucose levels before and after surgery, and the QOL scores evaluated 6 months after surgery.

Results The median (IQR) CRP, IL-6, and WBC values were significantly reduced in group PT on the first day compared with those in group C (12 (10-13.25) $\mathrm{mg} / \mathrm{l}, 10.45$ (9.575-11.65) pg/ml, 9 (7.75-9.25)×109\% I, vs 24 (20.75-26.25) mg/l, 25.35 (19.3-29.675) pg/ml, $12(11-13) \times 10^{9} / \mathrm{l}$, respectively, $\left.p<0.001\right)$. The median (IQR) QOL was significantly higher in group PT 6 months after surgery than in group C (53(51.75$55)$ vs 43 (41-51.5), $p<0.001)$. The incidence of chronic pain was significantly lower, $n / a l l$ was $4 / 33$ vs $0 / 34, p<0.001$.

Conclusions Ultrasound guided Pecs I, II and TTP block, significantly reduced the CRP, IL- 6 and WBC values on the first day; increased the QOL scores and the incidence of chronic pain 6 months after surgery; and promoted the rapid recovery of patients.

Trial registration: chictr.org.cn ChiCTR2000033275. Registered on 26 May 2020

\section{Introduction}

According to the 2018 global cancer statistics published by the American Cancer Society, $24.2 \%$ of the 8.6 million new cases of female cancer were breast cancer patients, ranking first in female cancer incidence. Approximately 630,000 women die of breast cancer every year (1). Surgical resection is the most important treatment for breast cancer at present. The wide incision easily causes a strong perioperative stress response and may lead to chronic pain and intractable pain after the operation. The incidence of persistent pain after breast cancer surgery (PPBCS) is $15-25 \%$, and some patients' postoperative pain lasts for several years (2). When ultrasound guided nerve block is applied during the perioperative period in breast cancer patients, it can significantly shorten the anesthesia time, reduce the amount of intraoperative infusion, reduce the incidence of postoperative complications, improve the comfort of patients, effectively promote the rehabilitation of patients, and improve the quality of life of 
patients with breast cancer. The aim of our current study was to evaluate the effect of the ultrasoundguided Pec $\nabla+P e c \nabla+T T P$ block in patients with MRM, the relevant indexes after the operation and the impact on the long-term quality of life of patients to provide a reference for the selection of an anesthesia scheme during the perioperative period in breast cancer patients.

The primary hypothesis was that the implementation of ultrasound-guided Pec $\nabla+P e c \nabla+$ TTP block would reduce the level of inflammatory factors and improve the long-term quality of life and the incidence of chronic pain.

\section{Materials And Methods}

This randomized, placebo-controlled and double-blind study was conducted at the Department of Anesthesiology at the First Affiliated Hospital of Guangxi Medical University, from 29 May 2020 to 23 Sep. 2020. The study was conducted according to the Consolidated Standards of Reporting Trials statement.

\section{Study design}

The inclusion criteria were participants with an American Society of Anesthesiologists class $\mathbb{Q} \square$, an age $\geq$ 18 years, a BMI $\leq 30 \mathrm{~kg} / \mathrm{m}^{2}$ and scheduled for MRM. The exclusion criteria were participants with an inability to cooperate, an allergy to local anesthetics (LA) or opioids, a local infection at the site of injection or systemic infection and Pecs $\nabla, \nabla$ and TTP block administration failure. One day before the operation, a routine preoperative visit was carried out to obtain the understanding and cooperation of patients, and all preoperative preparations were made. After entering the $\mathrm{OR}$, peripheral venous access was routinely achieved, and noninvasive blood pressure, pulse oxygen saturation and bispectral index (BIS) were monitored.

Anesthesia induction: intravenous injection of $0.05 \mathrm{mg} / \mathrm{kg}$ midazolam, $3 \mu \mathrm{g} / \mathrm{kg}$ sufentanil, $0.2 \mathrm{mg} / \mathrm{kg}$ etomidate, and $0.6 \mathrm{mg} / \mathrm{kg}$ rocuronium. After 2 minutes, tracheal intubation and mechanical ventilation were performed to control $\mathrm{PetCO}_{2}$ in the range of $35 \sim 45 \mathrm{mmHg}$.

Anesthesia maintenance: During the operation, target-controlled input (TCl) propofol and remifentanil to maintain a BIS of $40 \sim 55$ so that heart rate and blood pressure would not exceed $10 \%$ of the base value and rocuronium were administered intravenously at a single dose of $0.1-0.2 \mathrm{mg} / \mathrm{kg}$. Patients in both groups were given an intravenous patient-controlled analgesia (PCA) pump 15 minutes before the end of surgery. The PCA pump containing $1 \mu \mathrm{g} / \mathrm{ml}$ sufentanil with the following settings: background dose of 3 $\mathrm{ml} / \mathrm{h}$, press dose of $3 \mathrm{ml} / \mathrm{h}$, lockout time of $20 \mathrm{~min}$ and maximum of $10 \mathrm{ml} / \mathrm{h}$. Patients were instructed to administer a bolus whenever the numerical rating scale (NRS) pain scores exceeded $3 / 10$. All patients were transferred to the postanesthesia care unit (PACU) after surgery.

\section{Blinding}


Participants were randomly assigned to receive either active or placebo unilateral Pecs $\varangle, \varangle$, TTP block. The random number table method was used to ensure equal distribution in the two groups. A resident, not involved in the anesthesia of the study participants, would open the sealed opaque envelope containing the group allocation and (according to written instructions) prepare two $40-\mathrm{mL}$ syringes with either $40 \mathrm{~mL}$ of $0.25 \%$ ropivacaine or $0.9 \%$ saline. Thereafter, another resident controlled the procedure, and both signed the allocation paper before resealing the envelopes. The envelopes would only be opened by the investigators at the end of the study. Thus, the investigators, participants, care providers and those assessing outcomes were all blinded to the group allocation.

\section{Pecs $\llbracket, \bigotimes$, and TTP block procedure}

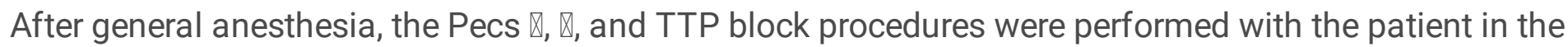
supine position with the arm abducted using an ultrasound unit (SonoSite M-Turbo) and a high frequency linear probe $(6 \sim 13 \mathrm{MHz})$. The skin was prepared with the application of iodophor and covered with a sterile hole towel. The ultrasound probe was covered with a sterile plastic sheath. Pecs $\otimes$ block (3): Using a similar probe position to that used when performing an infraclavicular brachial plexus block. Once the pectoralis major muscle (PMm) and pectoralis minor muscle (Pmm) had been identified, the location of the pectoral branch of the thoraco-acromial artery between the pectoralis muscles was checked with color Doppler. The lateral and median pectoral nerves were consistently located adjacent to the artery. A 22G, 100-mm needle (PAJUNK, UniPlex NanoLine, Germany) was infiltrated into the interfacial plane with 10 $\mathrm{ml}$ of $0.25 \%$ ropivacaine. Pecs $\otimes$ block (4): The ultrasound probe was placed at the midclavicular level inferolaterally to locate the axillary artery and vein and was then moved laterally until the lateral border of the Pmm and serratus anterior muscles (Sam) were identified at the level of the third rib. The 22G, 100$\mathrm{mm}$ needle was advanced in the potential space between the Pmm and Sam, and $20 \mathrm{ml}$ of $0.25 \%$ ropivacaine was deposited in this space. TTP block: The TTP block injection was administered as follows: $10 \mathrm{ml}$ of $0.25 \%$ ropivacaine was injected between the transversus thoracic muscle and the internal intercostal muscle between the third and fourth left ribs connecting at the sternum. The nerve block procedure described above and ultrasonic image processing were completed by the same skilled anesthesiologist (> 200 blocks).

\section{Outcome measures}

The primary outcomes were the changes in CRP, IL- 6 and WBC values on the first day before the operation and the first and third days after the operation, the changes in blood glucose levels before and after surgery, and the World Health Organization on QOL scores evaluated 6 months after the operation.

The secondary outcomes were the NRS pain scores of the patients $3,6,12,24$ and 48 hours after the operation, the operation time, the length of stay, the consumption of propofol and remifentanil during the operation, and the press times of the PCA pump at different time points after the operation; postoperative nausea and vomiting (PONV) was assessed using a four-point numerical scale $(0=$ no PONV, $1=$ mild nausea, 2 = severe nausea or vomiting once, and 3 = vomiting more than once), and adverse events were assessed including pneumothorax, vascular puncture, and local anesthetic toxicity. 
The QOL scores was used to investigate the patients six months after surgery, and the evaluation included appetite, mental state, sleep quality, physical activity, postoperative chronic pain, family understanding and support, and patients' attitudes toward treatment and daily life. The total score was 60: a score of $51 \sim 60$ was considered excellent, $41 \sim 50$ was good, $31 \sim 40$ was average, $21 \sim 30$ was poor, and $<20$ was extremely poor quality of life.

\section{Statistical analysis and sample size calculation}

IBM SPSS Statistics version 23 was used to test the normality of data by the Kolmogorov-Smirnov test. The normally distributed data were compared using Student's unpaired $t$ test, whereas nonparametric data were compared by the $\chi 2$ test or Fisher's exact test for intergroup differences. Variables are presented as the mean (SD), median (IQR), and range. The following variables were compared by using the Mann-Whitney U-test for pairwise comparisons: CRP, IL-6, WBC, and blood glucose values; NRS pain scores; length of stay; PONV numerical scale scores; and press times of the PCA pump.

Confidence intervals were calculated for statistically significant differences. The sample size was calculated on the basis of a pilot study. This pilot study revealed that the median (IQR) CRP and IL-6 levels were 22 (19.75-24.25) $\mathrm{mg} / \mathrm{l}$ and 22.35 (13.6-26.375) pg/ml, respectively, on the first day after the operation. For a difference in the $50 \%$ reduction of CRP and IL-6 levels on the first day after the operation at a significance level of 0.05 (two sided) and power of 0.8 , we required a minimum of 33 patients in each group. Assuming a $15 \%$ loss rate at the 6-month follow-up, we eventually enrolled 38 patients in each group.

\section{Results}

One hundred patients scheduled for MRM were screened for eligibility, and 76 participants were enrolled (Fig. 1). Two patients lost contact, five patients refused to conduct a questionnaire survey during followup, one patient developed tumor recurrence, and the other patient was seriously ill and died within 6 months after surgery. All the above patients were withdrawn from the follow-up study.

There were no significant differences in the CRP, IL-6, or WBC values between the two groups on the day before the operation. The median (IQR) CRP, IL-6, and WBC values were $24(20.75-26.25) \mathrm{mg} / \mathrm{l}, 25.35$ (19.3-29.675) pg/ml, and $12(11-13) \times 109 / /$, respectively, in group C vs 12 (10-13.25) mg/l, 10.45 (9.575$11.65) \mathrm{pg} / \mathrm{ml}$, and $9(7.75-9.25) \times 109 / \mathrm{l}$, respectively, in group PT, $p<0.001$, on the first day after the operation. On the third day after the operation, the WBC and IL- 6 values in the two groups recovered to normal levels before the operation, and there were no significant differences between the two groups. However, the median (IQR) CRP level was 11 (8-12.25) $\mathrm{mg} / \mathrm{l}$ in group C vs 4 (2.75-5) $\mathrm{mg} / \mathrm{l}$ in group PT, $p<$ 0.001. The median (IQR) blood glucose level at the end of the operation was $7.8(7.5-8.4) \mathrm{mmol} / \mathrm{l}$ in group C vs $5.4(5.3-5.8) \mathrm{mmol} / \mathrm{l}$ in group PT, $p<0.001$. The median (IQR) QOL score was $43(41-51.5)$ in group $C$ vs 53 (51.75-55) in group PT, $p<0.001$. The incidence of chronic pain/all, $\mathrm{n}$ was $4 / 33$ vs $0 / 34, p$ $<0.001$. (Table 1) 
The NRS pain scores (median (IQR)) were significantly lower in group PT at 0-48 hours: (1) at $3 \mathrm{~h}, 6 \mathrm{~h}, 12$ h, 24 h, and 48 h activity $1(0-1), 1(0-1), 1(1-2), 1(1-1), 1(1-1)$, respectively, vs $4(3-5), 4(3-5), 3$ (34), 3 (2-3), 2 (1-2), respectively, $p<0.001$; (2) at $3 \mathrm{~h}, 6 \mathrm{~h}, 12 \mathrm{~h}, 24 \mathrm{~h}, 48 \mathrm{~h}$ rest $0(0-0), 0(0-1), 0(0-1), 1$ $(1-1), 1(0-1)$, respectively, vs $3(2.75-4), 3(2.75-4), 3(2-3), 3(2-3), 1(1-2)$, respectively, $p<0.001$. (Table 2)

There was no significant difference in the operation time. The median (IQR) length of stay was $6(6-7)$ days in group $C$ vs $5(5-6)$ days in group PT, $p<0.001$. The mean (SD) consumption of propofol and remifentanil was 907.2 (51.04) $\mathrm{mg}$ and 1473.1 (242.5) $\mu \mathrm{g}$ in group C vs 517.1 (116.1) $\mathrm{mg}$ and 506.1 (212.8) $\mu \mathrm{g}$ in group PT, with mean differences of $390.1 \mathrm{mg}(95 \% \mathrm{Cl} 349.2$ to $431.1, p<0.001)$ and 967.2 $\mu \mathrm{g}(95 \% \mathrm{Cl} 862.9$ to $1071.4, p<0.001)$. The median (IQR) PONV score was $2(1-2)$ in group C vs $1(0-1)$ in group PT, $p<0.001$. (Table 2)

No adverse events, which included pneumothorax, vascular puncture, and local anesthetic toxicity, were recorded. 
Table 1. Patient characteristics, primary outcomes

\begin{tabular}{|c|c|c|c|c|}
\hline & Group C & Group PT & & $P$ value \\
\hline Patient characteristic & $46.8(7.4)$ & & $49.7(7.4)$ & 0.183 \\
\hline \multicolumn{5}{|l|}{ Age, mean (SD) } \\
\hline $\mathrm{BMI}, \mathrm{kg} / \mathrm{m}^{2}$ (SD) & $17.7(2.7)$ & \multicolumn{2}{|l|}{$16.9(1.7)$} & 0.215 \\
\hline Primary outcome & $3(2-4)$ & \multicolumn{2}{|l|}{$3(2-4)$} & 0.953 \\
\hline \multicolumn{5}{|l|}{ CRP, mg/I (median (IQR)) } \\
\hline \multicolumn{5}{|l|}{ Preoperative day } \\
\hline $1 \mathrm{~d}$ after operation & $24(20.75-26.25)$ & \multicolumn{2}{|l|}{$12(10-13.25)$} & $<0.001$ \\
\hline $3 \mathrm{~d}$ after operation & $11(8-12.25)$ & \multicolumn{2}{|l|}{$4(2.75-5)$} & $<0.001$ \\
\hline IL-6, pg/ml (median (IQR)) & $2(1.275-2.6)$ & \multicolumn{2}{|l|}{$2(1.275-2.6)$} & 1 \\
\hline Preoperative day & 25.35(19.3-29.675) & \multicolumn{2}{|c|}{ 10.45(9.575-11.65) } & $<0.001$ \\
\hline $1 \mathrm{~d}$ after operation & $3.9(2.55-4.525)$ & \multicolumn{2}{|l|}{$3.15(2.4-4.025)$} & 0.219 \\
\hline $3 \mathrm{~d}$ after operation & $6(5-7)$ & \multicolumn{2}{|l|}{$6(6-7)$} & 0.240 \\
\hline WBC, ×109/I (median (IQR)) & $12(11-13)$ & \multicolumn{2}{|l|}{$9(7.75-9.25)$} & $<0.001$ \\
\hline Preoperative day & $7(6-8)$ & \multicolumn{2}{|l|}{$7(6-8)$} & 0.392 \\
\hline \multicolumn{5}{|l|}{$1 \mathrm{~d}$ after operation } \\
\hline \multicolumn{5}{|l|}{$3 \mathrm{~d}$ after operation } \\
\hline \multicolumn{5}{|l|}{ Blood glucose, mmol/I (median (IQR)) } \\
\hline Before anesthesia induction & $5.2(5.1-5.425)$ & $5.2(5.0-5.325)$ & 0.318 & \\
\hline the end of operation & $7.8(7.5-8.4)$ & $5.4(5.3-5.8)$ & $<0.001$ & \\
\hline QOL scores (median (IQR)) & $43(41-51.5)$ & $53(51.75-55)$ & $<0.001$ & \\
\hline The incidence of chronic pain/all, $n$ & $4 / 33$ & $0 / 34$ & $<0.001$ & \\
\hline
\end{tabular}


Table 2. Secondary outcomes

\begin{tabular}{|c|c|c|c|}
\hline & Group C & Group PT & $P$ value \\
\hline NRS score & $4(3-5)$ & $1(0-1) 1(0-1)$ & $<0.001<0.001$ \\
\hline \multicolumn{4}{|l|}{ median at $3 \mathrm{~h}$ activity (IQR) } \\
\hline median at $6 \mathrm{~h}$ activity (IQR) & $4(3-4)$ & $1(0-1)$ & $<0.001$ \\
\hline median at $12 \mathrm{~h}$ activity (IQR) & $3(3-4)$ & $1(1-2)$ & $<0.001$ \\
\hline median at $24 \mathrm{~h}$ activity (IQR) & $3(2-3)$ & $1(1-1)$ & $<0.001$ \\
\hline median at $48 \mathrm{~h}$ activity (IQR) & $2(1-2)$ & $1(1-1)$ & $<0.001$ \\
\hline median at $3 \mathrm{~h}$ rest (IQR) & $3(2.75-4)$ & $0(0-0)$ & $<0.001$ \\
\hline median at $6 \mathrm{~h}$ rest (IQR) & $3(2.75-4)$ & $0(0-1)$ & $<0.001$ \\
\hline median at $12 \mathrm{~h}$ rest (IQR) & $3(2-3)$ & $0(0-1)$ & $<0.001$ \\
\hline median at $24 \mathrm{~h}$ rest (IQR) & $3(2-3)$ & $1(1-1)$ & $<0.001$ \\
\hline median at $48 \mathrm{~h}$ rest (IQR) & $1(1-2)$ & $1(0-1)$ & $<0.001$ \\
\hline operation time, min (mean (SD)) & 137.0(37.3) & 132.2(46.3) & 0.115 \\
\hline median length of stay (IQR) & $6(6-7)$ & $5(5-6)$ & $<0.001$ \\
\hline consumption of propofol, mg (mean (SD)) & $907.2(51.04)$ & $517.1(116.1)$ & $<0.001$ \\
\hline consumption of remifentanil, $\mu \mathrm{g}$ (mean (SD)) & $1473.1(242.5)$ & $506.1(212.8)$ & $<0.001$ \\
\hline press times of PCA pump at $0-24$ hours & $13(12-15)$ & $5(3-6)$ & $<0.001$ \\
\hline median (IQR) & $14(13-15)$ & $6(5-8)$ & $<0.001$ \\
\hline press times of PCA pump at $24-48$ hours & $2(1-2)$ & $1(0-1)$ & $<0.001$ \\
\hline \multicolumn{4}{|l|}{ median (IQR) } \\
\hline median PONV score (IQR) & & & \\
\hline
\end{tabular}

\section{Discussion}

As is well known, MRM is associated with a large incision range, high intraoperative stress, a high incidence of postoperative acute pain, and long-term development of chronic pathological pain. Regional block has been used to optimize perioperative analgesia management, reduce the consumption of opioids, and promote the recovery of gastrointestinal function, and early postoperative functional exercise has been conducive to the rehabilitation of patients. This management mode has achieved remarkable results in the operation of patients with liver cancer (5), gastric cancer (6) and colorectal cancer (7). It can effectively reduce the stress response of patients during the perioperative period, 
accelerate the recovery of patients, reduce the length of hospital stay, and reduce the economic burden of patients.

At present, most studies focus on reducing the consumption of opioids and the postoperative pain scores of breast cancer patients after MRM by Pecs nerve block or thoracic paravertebral block (8). However, there are few studies on the objective evaluation of the perioperative stress response by some stressrelated serological indicators and on the long-term follow-up evaluation of postoperative quality of life and the incidence of chronic pain.

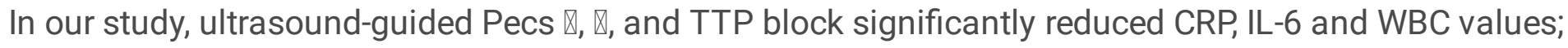
significantly increased the QOL scale scores and the incidence of chronic pain 6 months after the operation; significantly reduced the consumption of opioids during and after the operation; significantly reduced the NRS pain scores at each time point after the operation; significantly reduced the PONV scores; shortened the length of stay; and promoted the rapid recovery of patients.

Since Blanco et al. proposed a series of novel types of nerve block, Pecs $\otimes(3)$, Pecs $\otimes(9)$, and anterior serratus plane block (10), these new techniques have been widely used in clinical practice. With the

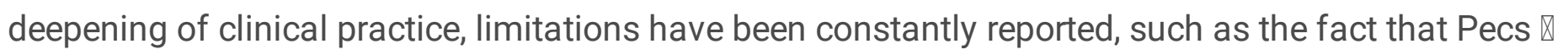
block is not able to block the anterior branches of those nerves (2nd $\sim 6$ th), which collect the sensory innervation of the medial part of the breast (11). Ueshima (12) et al. proposed the TTP, which can effectively block the medial breast region. Our study confirmed that the intraoperative consumption of

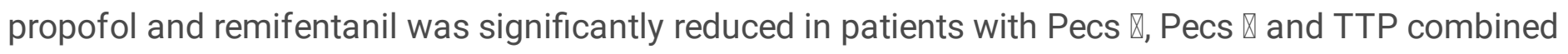
with general anesthesia, the need for analgesics was reduced within 48 hours after surgery, and the length of stay was shorter, which was consistent with some studies (13). In addition, we also observed that the NRS pain score in group PT was lower than that in group C. However, the duration of the analgesic effect was much longer than that of local anesthetics. We considered that the regional block may be associated with the reduction of peripheral and central pain sensitization due to lower levels of inflammation and a better immune response (14). The CRP, IL- 6 and WBC values in group PT were significantly lower than those in group $\mathrm{C}$ on the first day after the operation, and the CRP level was still

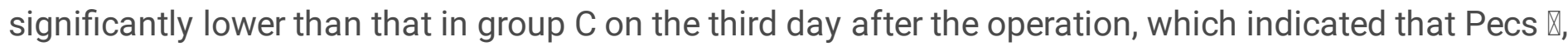
Pecs $\nabla$ and TTP block could effectively reduce the stress response of MRM, even lasting until the third day after operation. This may partly explain why the NRS score $48 \mathrm{~h}$ after the operation was still lower than that in group $\mathrm{C}$.

It has been the common goal of all doctors to promote the rapid recovery of cancer patients. Rapid recovery should not only reduce the pain of patients but also reduce the hospitalization expenses of patients, which is very important for both patients and medical insurance bureau. Moreover, it should improve the long-term quality of life of patients and reduce the recurrence and metastasis of cancer. Our study also has some limitations: the QOL scores evaluation was only conducted 6 months after surgery, and no longer follow-up evaluations were conducted. In the future, we will carry out a longer follow-up evaluation to observe the QOL scores of these patients. It has been reported that regional block can 
reduce the recurrence and metastasis of tumor patients. However, this idea is still controversial, and more multicenter studies are needed to eliminate multiple interfering factors. Therefore, we plan to continue to observe whether the Pecs $\nabla, P e c s \rrbracket$ and TTP block, is associated with cancer recurrence and metastasis in breast cancer patients postoperatively.

\section{Conclusions}

Ultrasound-guided Pecs $\varangle, \varangle$, and TTP block significantly reduced the CRP, IL- 6 and WBC values; significantly increased the QOL scores and the incidence of chronic pain 6 months after the operation; significantly reduced the consumption of opioids during and after the operation; significantly reduced the NRS pain scores at each time point after the operation; significantly reduced the PONV scores; shortened the length of stay; and promoted the rapid recovery of patients.

\section{Abbreviations}

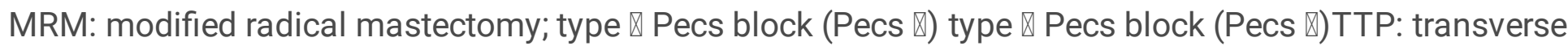
thoracic muscle plane block; QOL: Quality of life brief scale; PPBCS: persistent pain after breast cancer surgery; LA: local anesthetics; BIS: bispectral index; TCl: target-controlled input; PCA: patient-controlled analgesia; NRS: numerical rating scale; PACU: postanesthesia care unit; PMm: pectoralis major muscle; Pmm: pectoralis minor muscle; Sam: serratus anterior muscles; PONV: postoperative nausea and vomiting;

\section{Declarations}

\section{Ethics approval and consent to participate}

Ethical approval was obtained from the ethics committee of the First Affiliated Hospital of Guangxi Medical University (KY-E-093). Written informed consent was obtained from all participants before enrolling for the study. No data from participants are reported.

\section{Consent for publication}

Not applicable.

\section{Availability of data and materials}

The datasets generated and/or analysed during the current study are held by the First Affiliated Hospital of Guangxi Medical University. Any requests for anonoymised data should be made to First Affiliated Hospital of Guangxi Medical University

\section{Competing interests}

The authors declare that they have no competing interests. 


\section{Funding}

This work was supported by the National Key Research and Development Program of China (Grant No.2018YFC2001905) and Guangxi Key Research and Development Program (No. AB20159019) and the Health Committee of Guangxi Zhuang Autonomous Region (S2017039, Z20190252).

\section{Authors' contributions}

TL, LC, YX are the Chief Investigator; they conceived the study, led the proposal and protocol development. MS, WD contributed to study design and to development of the proposal. JC was the lead trial methodologist. All authors read and approved the final manuscript.

\section{Acknowledgements}

Not applicable.

\section{References}

1. Bray F, Ferlay J, Soerjomataram I, Siegel RL, Torre LA, Jemal A. Global cancer statistics 2018 : GLOBOCAN estimates of incidence and mortality worldwide for 36 cancers in 185 countries. Cancer J Clin. 2018;68(6):394-424.

2. Waltho D, Rockwell G. Post-breast surgery pain syndrome: establishing a consensus for the definition of post-mastectomy pain syndrome to provide a standardized clinical and research approach - a review of the literature and discussion. Canadian journal of surgery Journal canadien de chirurgie. 2016;59(5):342-50.

3. Blanco R. The 'pecs block': a novel technique for providing analgesia after breast surgery. Anaesthesia. 2011;66(9):847-8.

4. Kulhari S, Bharti N, Bala I, Arora S, Singh G. Efficacy of pectoral nerve block versus thoracic paravertebral block for postoperative analgesia after radical mastectomy: a randomized controlled trial. Br J Anaesth. 2016;117(3):382-6.

5. Blanco R, Fajardo M, Parras Maldonado T. Ultrasound description of Pecs II (modified Pecs I): a novel approach to breast surgery. Revista espanola de anestesiologia y reanimacion. 2012;59(9):470-5.

6. Ratti F, Cipriani F, Reineke R, Catena M, Comotti L, Beretta L, et al. Impact of ERAS approach and minimally-invasive techniques on outcome of patients undergoing liver surgery for hepatocellular carcinoma. Digestive and liver disease: official journal of the Italian Society of Gastroenterology and the Italian Association for the Study of the Liver. 2016;48(10):1243-8.

7. Hughes MJ, Chong J, Harrison E, Wigmore S. Short-term outcomes after liver resection for malignant and benign disease in the age of ERAS. HPB: the official journal of the International Hepato Pancreato Biliary Association. 2016;18(2):177-82.

8. Wee IJY, Syn NL, Shabbir A, Kim G, So JBY. Enhanced recovery versus conventional care in gastric cancer surgery: a meta-analysis of randomized and non-randomized controlled trials. Gastric cancer: 
official journal of the International Gastric Cancer Association the Japanese Gastric Cancer Association. 2019;22(3):423-34.

9. Aoyama T, Yoshikawa T, Sato T, Hayashi T, Yamada T, Ogata T, et al. Equivalent feasibility and safety of perioperative care by ERAS in open and laparoscopy-assisted distal gastrectomy for gastric cancer: a single-institution ancillary study using the patient cohort enrolled in the JCOG0912 phase III trial. Gastric cancer: official journal of the International Gastric Cancer Association the Japanese Gastric Cancer Association. 2019;22(3):617-23.

10. Gustafsson UO, Scott MJ, Hubner M, Nygren J, Demartines N, Francis N, et al. Guidelines for Perioperative Care in Elective Colorectal Surgery: Enhanced Recovery After Surgery (ERAS(®)) Society Recommendations: 2018. World journal of surgery. 2019;43(3):659-95.

11. van Rooijen S, Carli F, Dalton S, Thomas G, Bojesen R, Le Guen M, et al. Multimodal prehabilitation in colorectal cancer patients to improve functional capacity and reduce postoperative complications: the first international randomized controlled trial for multimodal prehabilitation. BMC Cancer. 2019;19(1):98.

12. Senapathi TGA, Widnyana IMG, Aribawa I, Jaya A, Junaedi IMD. Combined ultrasound-guided Pecs II block and general anesthesia are effective for reducing pain from modified radical mastectomy. Journal of pain research. 2019;12:1353-8.

13. Blanco R, Parras T, McDonnell JG, Prats-Galino A. Serratus plane block: a novel ultrasound-guided thoracic wall nerve block. Anaesthesia. 2013;68(11):1107-13.

14. Sopena-Zubiria LA, Cuéllar-Martínez A, Galán Gutiérrez JC, Fernandez-Meré LA. Reply to the article entitled "Ultrasound description of Pecs II (modified Pecs I): a novel approach to breast surgery". Revista espanola de anestesiologia y reanimacion. 2013;60(5):295.

15. Ueshima H, Kitamura A. Blocking of Multiple Anterior Branches of Intercostal Nerves (Th2-6) Using a Transversus Thoracic Muscle Plane Block. Regional anesthesia pain medicine. 2015;40(4):388.

16. Ueshima $\mathrm{H}$, Hara $E$, Marui T, Otake $H$. The ultrasound-guided transversus thoracic muscle plane block is effective for the median sternotomy. J Clin Anesth. 2016;29:83.

17. Ueshima H, Kimura S, Otake H. Bilateral breast cancer resection performed under the bilateral transversus thoracic muscle plane block. J Clin Anesth. 2016;33:413.

18. Bashandy GM, Abbas DN. Pectoral nerves I and II blocks in multimodal analgesia for breast cancer surgery: a randomized clinical trial. Regional anesthesia pain medicine. 2015;40(1):68-74.

19. Barrington MJ, Seah GJ, Gotmaker R, Lim D, Byrne K. Quality of Recovery After Breast Surgery: A Multicenter Randomized Clinical Trial Comparing Pectoral Nerves Interfascial Plane (Pectoral Nerves II) Block With Surgical Infiltration. Anesthesia analgesia. 2020;130(6):1559-67.

20. Pérez-González O, Cuéllar-Guzmán LF, Soliz J, Cata JP. Impact of Regional Anesthesia on Recurrence, Metastasis, and Immune Response in Breast Cancer Surgery: A Systematic Review of the Literature. Regional anesthesia pain medicine. 2017;42(6):751-6.

\section{Figures}




\section{$\Rightarrow$ CONSORT \\ TRANSPARENT REPORTING of TRIALS}

\section{CONSORT 2010 Flow Diagram}

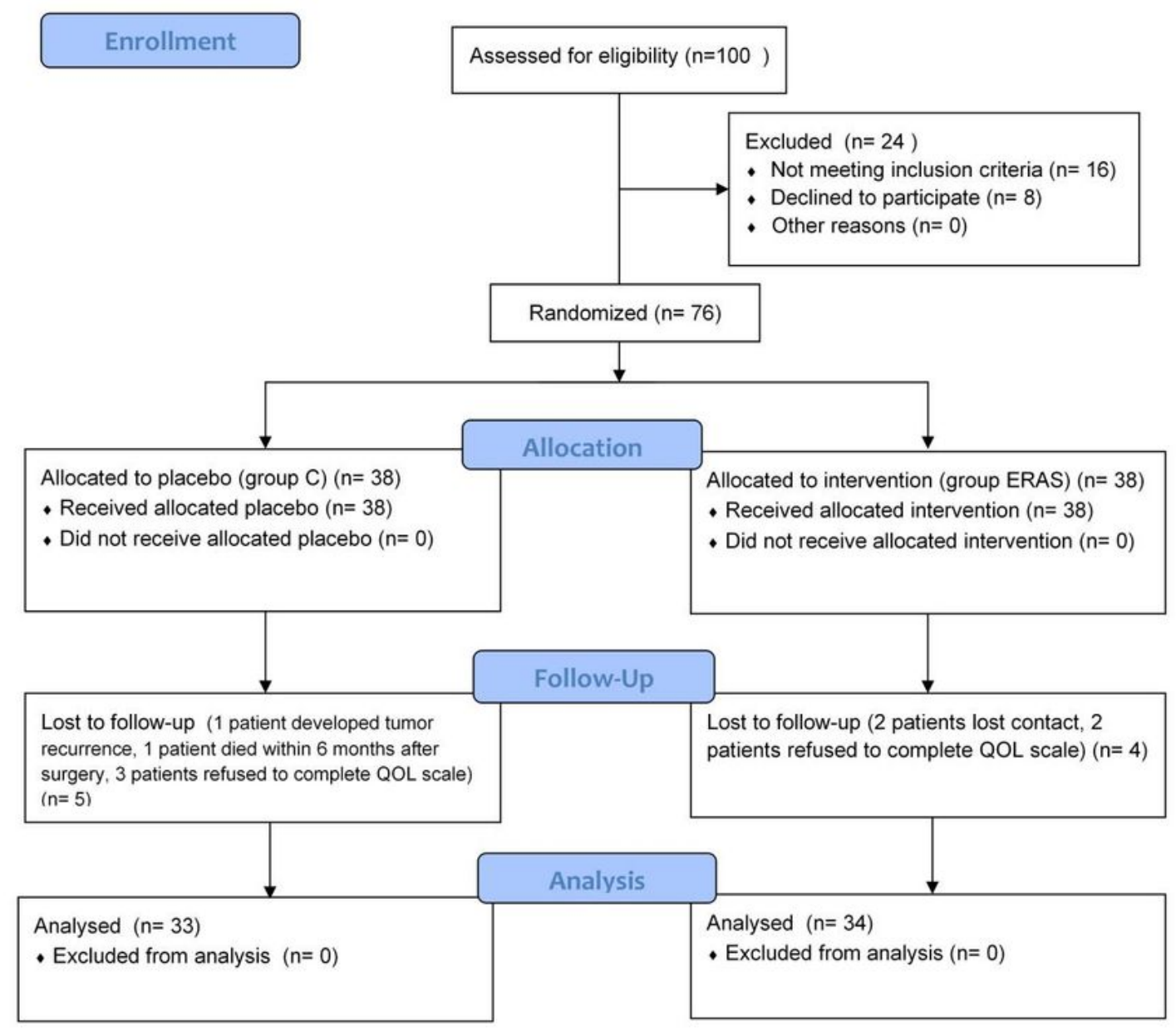

\section{Figure 1}

Consolidated Standards of Reporting Trials (CONSORT) flow diagram. 